\title{
SOLVENT EXTRACTION OF HYDROCHLORIC ACID BY LONG-CHAIN ALKYLAMINE IN A STIRRED TRANSFER CELL
}

\author{
TERUO TSUNEYUKI, KAZUO KONDO, YoSHINOBU KAWANO \\ AND FUMIYUKI NAKASHIO \\ Department of Organic Synthesis, Kyushu University, \\ Fukuoka 812
}

\begin{abstract}
Authors indicated previously that the interfacial rate process was significant in low concentration range of long-chain alkylamine for the extraction of hydrochloric acid in a horizontal rectangular channel contactor. This paper is concerned with the effects of interfacial rate process for the same extraction system in a stirred transfer cell.

It was found from the analysis of the initial rate of extraction that the interfacial rate process was significant in the range of concentration of long-chain alkylamine lower than $0.1 \mathrm{~mol} / l$, while in the range of concentration of the amine higher than $0.2 \mathrm{~mol} / l$ the interfacial rate process was assumed to be an instantaneous irreversible reaction.

These experimental results were interpreted by the diffusion model, taking account of the interfacial rate process expressed by $(1,2)$ order irreversible reaction with respect to the interfacial concentrations of hydrochloric acid and long-chain alkylamine.
\end{abstract}

\section{Introduction}

Liquid surfactant membranes containing mobile carriers, such as long-chain alkylamine, macrocyclic polyether or chelating agents, have attracted special interest recently, as they offer a new and effective method to separate and to concentrate heavy metal ions from waste-water ${ }^{11}$. These processes are generally performed at low concentration of carriers because they are rather expensive. Long-chain alkylamine has been used to extract heavy metal ions or acids also by conventional means ${ }^{5}$. To investigate the extraction mechanism of acids by long-chain alkylamine the extraction rate has been measured using various equipment, such as a diffusion cell' ${ }^{2}$, a laminar jet apparatus ${ }^{7}$, a stirred transfer cell $^{6}$, and a single-drop method ${ }^{4}$. Many of these investigations were carried out at rather high concentration of long-chain alkylamine, where the rate of extraction was mostly assumed to be controlled by the diffusion near the interface. There have been few references concerned with investigation in low concentration ranges where a separation process utilizing liquid surfactant membranes is performed.

Authors indicated in the previous paper ${ }^{8)}$ that an interfacial rate process is significant in low concentration ranges of long-chain alkylamine and hydro-

Received September 19, 1977. Correspondence concerning this article should be addressed to F. Nakashio. T. Tsuneyuki is now with Kitakyushu Technical College, Shii, Kitakyushu 803, Y. Kawano is now with Miyazaki Univ., Miyazaki 880. chloric acid.

This paper concerns the effects of interfacial rate process on the extraction rate of hydrochloric acid by long-chain alkylamine using a stirred transfer cell, in which the flow pattern near the interface was different from that of the horizontal rectangular channel contactor described in the previous paper.

\section{Theory}

Since the reaction between hydrochloric acid and long-chain alkylamine is rapid and the solubility of long-chain alkylamine in aqueous solution is very small, it follows that amine salt is likely to be formed near or at the interface between aqueous and organic phases. Furthermore, it was found from the measurement of interfacial tension ${ }^{3}$ ' that long-chain alkylamine and its salt behave like surface-active agent in that they may be concentrated at the interface by adsorption. As a result, the hydrochloric acid extracted encounters three separate resistances, due to diffusion through the aqueous phase, across the interface and through the organic phase, respectively. The interfacial resistance may include the adsorption of long-chain alkylamine to the interface, the reaction between hydrochloric acid and adsorbed long-chain alkylamine to form amine salt, and desorption of amine salt from the interface. Although the precise rate equation for these interfacial rate processes are quite complicated and difficult to obtain, it is considered to be expressed as 


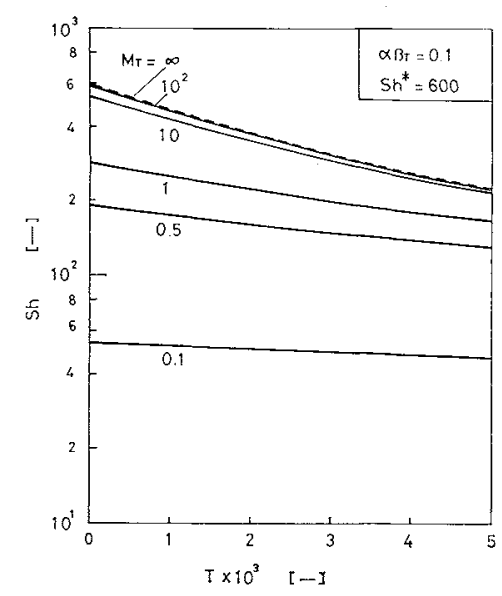

(a) $\alpha \beta_{T}=0.1$

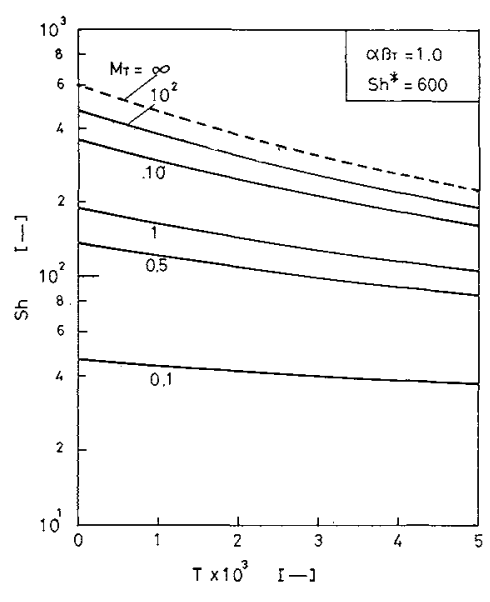

(b) $\alpha \beta_{T}=1.0$

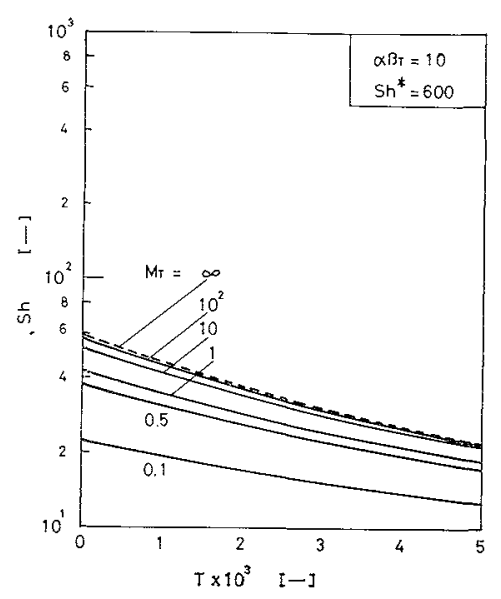

(c) $\alpha \beta_{T}=10$

Fig. $1 S h$ as a function of $T$

$$
R=k_{r} C_{A i}^{m} C_{B i}^{n}
$$

where $C_{A i}$ and $C_{B i}$ are the concentrations of hydrochloric acid in the aqueous phase adjoining the interface and that of long-chain alkylamine in the organic phase adjoining the interface, $m$ and $n$ are the apparent orders of the interfacial reaction and $k_{r}$ is the rate constant.

Since the extraction rate in a stirred transfer cell is rather slow, a pseudo-steady state will be reached in each instance; then the extraction rate of hydrochloric acid can be expressed as

$$
N_{A}=k_{A}\left(C_{A}-C_{A i}\right)=k_{B}\left(C_{B}-C_{B i}\right)=k_{r} C_{A i}^{m} C_{B i}^{n}
$$

where $k_{A}$ and $k_{B}$ are the mass transfer coefficients in each phase. The following equations are obtained from the mass balance in a stirred transfer cell:

$$
\begin{aligned}
& -V_{A} \frac{d C_{A}}{d t}=k_{A} S\left(C_{A}-C_{A i}\right) \\
& -V_{B} \frac{d C_{B}}{d t}=k_{B} S\left(C_{B}-C_{B i}\right)
\end{aligned}
$$

where $V_{A}$ and $V_{B}$ are the volumes of each phase and $S$ is the interfacial area. The basic equations, (2), (3a) and (3b), are numerically solved to obtain a timecourse of the concentrations of hydrochloric acid and long-chain alkylamine when the apparent orders of the interfacial reaction, the reaction rate constant, mass transfer coefficients in each phase, the volumes of each phase, and the interfacial area are given.

The basic equations are rewritten using dimensionless variables to compare them with the theoretical results for the horizontal rectangular channel contactor reported in the previous paper ${ }^{8}$.

$$
\begin{aligned}
& Y_{A}=Y_{A i}+\alpha^{m-1} M_{T} Y_{A i}^{m} Y_{B i}^{n} \\
& Y_{B}=Y_{B i}+\alpha^{m} \beta_{T} M_{T} Y_{A i}^{m} Y_{B i}^{n} \\
& -\frac{d Y_{A}}{d T}=S h^{*}\left(Y_{A}-Y_{A i}\right)
\end{aligned}
$$

and $Y_{B}$ is also expressed as

$$
Y_{B}=1-\alpha v\left(1-Y_{A}\right)
$$

where the dimensionless variables are

$$
\begin{aligned}
& Y_{A}=C_{A} / C_{A 0} \quad Y_{A i}=C_{A i} / C_{A 0} \\
& Y_{B}=C_{B} / C_{B 0} \quad Y_{B i}=C_{B i} / C_{B 0} \\
& \alpha=C_{A 0} / C_{B 0} \quad \beta_{T}=k_{A} / k_{B} \\
& M_{T}=k_{r} C_{B 0}^{m+n-1} / k_{A} \quad T=\left(S / V_{A}\right)^{2} D_{A} t \\
& S h^{*}=\left(V_{A} / S\right) k_{A} / D_{A} \quad v=V_{A} / V_{B}
\end{aligned}
$$

As before, Eqs. (4)-(7) are numerically solved to obtain the dimensionless average extraction rate, $S h$, from the following equation:

$$
S h=\frac{S h^{*}}{T} \int_{0}^{T}\left(Y_{A}-Y_{A i}\right) d T
$$

When the interfacial reaction rate is assumed to be instantaneous, that is, $M_{T}=\infty, Y_{A i}$ can be considered negligibly smaller than $Y_{A}$ in the case of $\alpha \beta_{T}<1$. In this case, Eq. (6) can be analytically integrated with $Y_{A i}=0$, and then Eq. (9) also can be integrated to obtain the expression for $S h$. When $M_{T^{r}}=\infty$ and $\alpha \beta_{T}>1, Y_{B i}$ can be considered negligibly smaller than $Y_{B}$. Therefore, a mathematical manipulation with respect to $Y_{B}$ and $Y_{B}$, in a similar manner as for $Y_{A}$ and $Y_{A i}$, leads to the expression for $S h$. These are as follows:

$$
\begin{array}{ll}
S h=(1 / T)\left\{1-\exp \left(-S h^{*} T\right)\right\} & \left(\alpha \beta_{T}<1\right) \\
S h=(1 / \alpha T)\left[1-\exp \left\{-\left(S h^{*} / \beta_{T}\right) T\right\}\right] & \left(\alpha \beta_{T}>1\right)
\end{array}
$$

It was shown in the previous paper that $m=1, n=2$ and $k_{r}=5.4 \times 10^{6} \mathrm{~cm}^{7} / \mathrm{mol}^{2} \cdot \mathrm{sec}$ for the extraction of hydrochloric acid by long-chain alkylamine in the rectangular channel contactor. The numerical analysis was done by using these values, and the results are shown as full lines in Fig. 1. The dotted lines in Fig. 1 are the calculated values from Eqs. (10) and (11), where the extraction rate is controlled only by the diffusion step in the bulk phase. Figure 1 includes the case of $\alpha \beta_{T}=0.1$, where the interfacial concentration $Y_{A i}$ is negligibly small, and the case $\alpha \beta_{T}=10$, where 


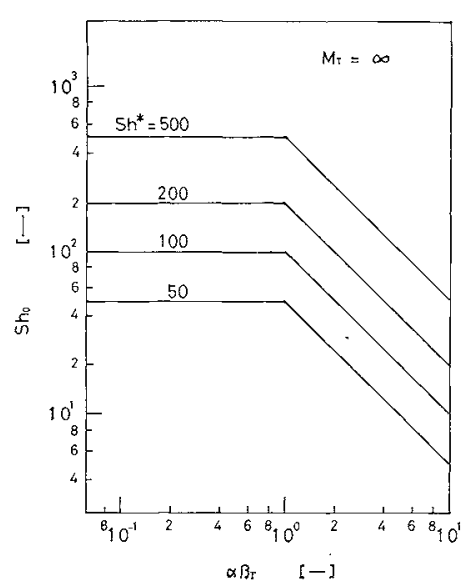

Fig. 2 Effect of $S h^{*}$ on $S h_{0}$

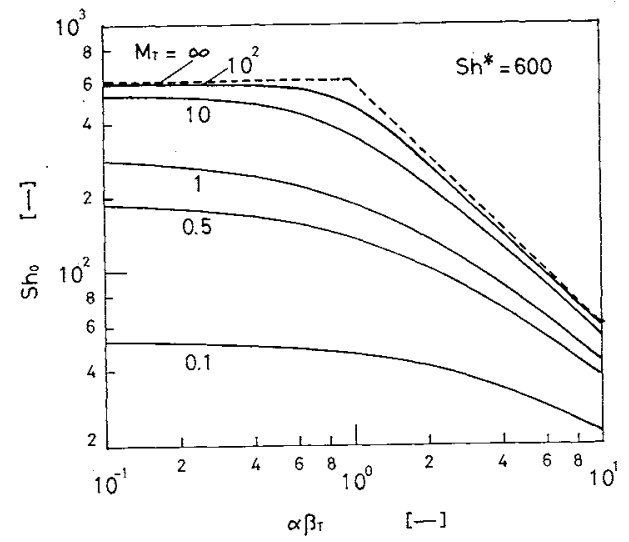

Fig. 3 Relationship between $S h_{0}$ and $\alpha \boldsymbol{\beta}_{T}$

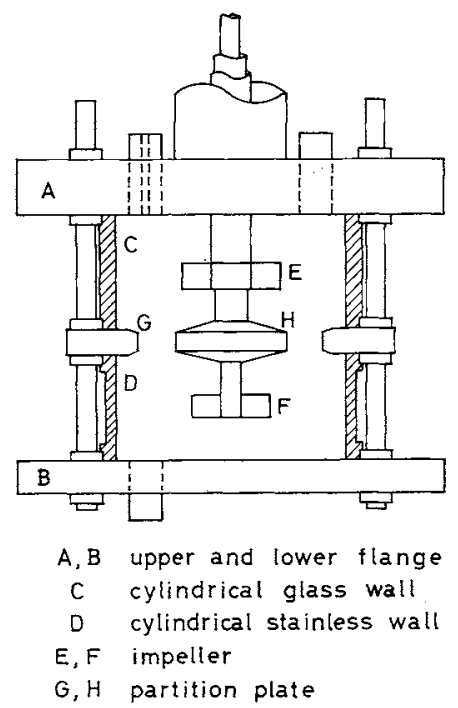

Fig. 4 Experimental apparatus

$Y_{A i}$ is fairly large and cannot be disregarded. In Fig. 2, the effect of the stoichiometric parameter, $\alpha \beta_{T}$, on the initial extraction rate, $S h_{0}$, is shown for the case of $M_{T}=\infty$. These relationships between $S h_{0}$ and $\alpha \beta_{T}$ are given for $T \rightarrow 0$ from Eqs. (10) and (11) as follows:

$$
\begin{array}{ll}
S h_{0}=S h^{*} & \left(\alpha \beta_{T}<1\right) \\
S h_{0}=S h^{*} / \alpha \beta_{T} & \left(\alpha \beta_{T}>1\right)
\end{array}
$$

$S h_{0}$ at $M_{T}=\infty$ corresponds to the maximum extraction rate at a certain $S h^{*}$, which is specified by the hydrodynamics near the interface.

Since $S h$ varies gradually with $T$ as shown in Fig. 1, the experimental results are analysed only for the initial rate of extraction. Figure 3 shows the relationship between $S h_{0}$ and $\alpha \beta_{T}$, which were obtained numerically from the basic equations for various values of $M_{T}$. It is seen that $S h_{0}$ is nearly equal to $S h^{*}$ in the case of $M_{T}>10^{2}$, except for the range of $3>\alpha \beta_{T}>0.5$, and that $S h_{0}$ decreases with increase of $\alpha \beta_{T}$, especially in the range of $\alpha \beta_{T}>1$.

\section{Apparatus and Method of Experiment}

The stirred transfer cell used is shown in Fig. 4, and it is similar to that of Lewis ${ }^{9}$, but is slightly modified. The inner diameter of the vessel was $70 \mathrm{~mm}$ and the length of the stirrer blades was $30 \mathrm{~mm}$ for the upper phase and $25 \mathrm{~mm}$ for the lower one. All metal parts in contact with reagent solutions were made of stainless steel. An electric conductivity cell was installed in the lower part of the vessel to follow the concentration change of hydrochloric acid in the aqueous phase. The transfer cell was situated within a bath through which water maintained at $30.0 \pm 0.1^{\circ} \mathrm{C}$ could be circulated.

Aqueous solutions were prepared by diluting commercial GR grade hydrochloric acid with ion-exchanged water. Amberlite LA-2 (commercial name of $N$-lauryl-(trialkylmethyl) amine, shortened to amine hereinafter) was used as the long-chain alkylamine. Amine was first purified by the same method as described elsewhere ${ }^{10)}$, and then was diluted with commercial GR grade $n$-hexane to the required concentration.

Aqueous phase was first introduced to the transfer cell from a buret fitted with constant temperature jacket, and then the organic solution was placed carefully in such a way as not to disturb the interface. The two stirrers were driven independently with two motors, and the stirring speeds were checked with a stroboscope.

\section{Results and Discussion}

Provided that the interfacial rate process is approximated by $(m, n)$ order irreversible reaction respect to the interfacial concentrations of hydrochloric acid and amine, the dimensionless coefficient of average extraction rate of hydrochloric acid $S h\left(=N_{A} V_{A} / S D_{A} C_{A 0}\right)$ is related to the ratio of initial concentrations $\alpha\left(=C_{A 0}\right.$ / $C_{B 0}$ ), the ratio of mass transfer coefficients at each phase $\beta_{T}\left(=k_{A} / k_{B}\right)$ and $M_{T}\left(=k_{r} C_{B 0}^{m+n-1} / k_{A}\right)$ which contains the interfacial reaction rate constant and the concentration of amine. Therefore, the extraction 


\begin{tabular}{|c|c|c|c|c|c|}
\hline Run & $\begin{array}{c}C_{A 0} \times 10^{3} \\
{\left[\mathrm{~mol} / \mathrm{cm}^{3}\right]}\end{array}$ & $\begin{array}{c}\mathrm{C}_{B 0} \times 10^{3} \\
{\left[\mathrm{~mol} / \mathrm{cm}^{3}\right]}\end{array}$ & {$[\stackrel{\alpha}{-}]$} & $\begin{array}{l}S h_{0} \\
{[--]}\end{array}$ & $\begin{array}{l}M_{T} \\
{[-]}\end{array}$ \\
\hline 5 & 0.0200 & 0.0119 & 1.68 & 98 & 0.25 \\
\hline 1 & 0.0100 & $"$ & 0.84 & 131 & $"$ \\
\hline 2 & $"$ & " & $"$ & 124 & $"$ \\
\hline 6 & 0.0200 & 0.0170 & 1.18 & 137 & 0.50 \\
\hline 10 & $"$ & $"$ & " & $"$ & $n$ \\
\hline 11 & 0.0140 & $" \prime$ & 0.82 & 146 & " \\
\hline 7 & 0.0100 & $"$ & 0.59 & 141 & $n$ \\
\hline 16 & $" \prime$ & $"$ & $"$ & 182 & $" 1$ \\
\hline 18 & 0.0326 & 0.0300 & 1.09 & 215 & 1.6 \\
\hline 17 & 0.0202 & $"$ & 0.68 & 254 & $"$ \\
\hline 20 & 0.0142 & $"$ & 0.47 & 254 & $n$ \\
\hline 19 & 0.0101 & $"$ & 0.34 & 281 & $"$ \\
\hline 21 & 0.1009 & 0.0911 & 1.11 & 250 & 15 \\
\hline 22 & 0.0571 & " & 0.63 & 333 & $"$ \\
\hline 25 & $"$ & " & $"$ & 346 & $"$ \\
\hline 29 & 0.0326 & " & 0.36 & 422 & $"$ \\
\hline 24 & 0.0163 & $"$ & 0.179 & 422 & $" \prime$ \\
\hline 28 & 0.1009 & 0.201 & 0.50 & 471 & 70 \\
\hline 26 & 0.0326 & " & 0.162 & 602 & $"$ \\
\hline 27 & 0.0163 & $"$ & 0.081 & 576 & " \\
\hline 30 & 0.0790 & 0.344 & 0.24 & 580 & 194 \\
\hline
\end{tabular}

rate in a stirred transfer cell was measured with various $\alpha$ and $C_{B 0}$, as shown in Table 1. All the experiments were carried out at a stirring speed of $80 \mathrm{rpm}$.

Figure 5 shows the typical results of time-course of acid concentration. The tangents of these curves correspond to the rate of extraction. It can be seen from Fig. 5 that in the case of $C_{B 0}<0.1 \times 10^{-3}$ the extraction rate varies with either $\alpha$ or $C_{B 0}$ : even at equal $C_{B 0}$ the extraction rate increases with decrease of $\alpha$, and even at nearly equal $\alpha$ the extraction rate increases with $C_{B 0}$. In the case of $C_{B 0}>0.2 \times 10^{-3}$, however, the initial rates of extraction were scarcely affected by $\alpha$ and $C_{B 0}$, as shown in Fig. 5. It was assumed that these were the upper limit of the extraction rate, which corresponds to the line with $M_{T}=\infty$ in Fig. 3. Therefore, $S h^{*}$ was determined from these data to be 600 , and then $k_{A}$ was calculated as $3.0 \times 10^{-3} \mathrm{~cm} / \mathrm{sec}$ from Eq. (8).

The values of $S h_{0}$ obtained by experiments and the results of numerical analysis based on the abovementioned mathematical model are superimposed in Fig. 6 with $S h^{*}=600$. Although $\beta_{T}$ is to be obtained virtually as the ratio of the mass transfer coefficients in each phase, as defined by Eq. (8), it was calculated assuming either $\beta_{T}=\sqrt{D_{A}} / \bar{D}_{B}$ or $\beta_{T}=D_{A} / D_{B}$ because $k_{B}$ could not be determined experimentally. The values of $D_{A}$ and $D_{B}$ used were $3.87 \times 10^{-5} \mathrm{~cm}^{2} / \mathrm{sec}^{1)}$ and $1.41 \times 10^{-5} \mathrm{~cm}^{2} / \mathrm{sec}$, respectively, and $D_{B}$ was measured by capillary-cell method ${ }^{12)}$. Since $m, n$ and $k_{r}$ had been obtained in a rectangular channel contactor as shown previously in this paper, the values of $M_{T}$ were calculated from Eq. (8) and are shown in Table 1. From Figs. 6 (a) and 6 (b), the experimental results are more likely to agree with the diffusion model that takes account of the interfacial

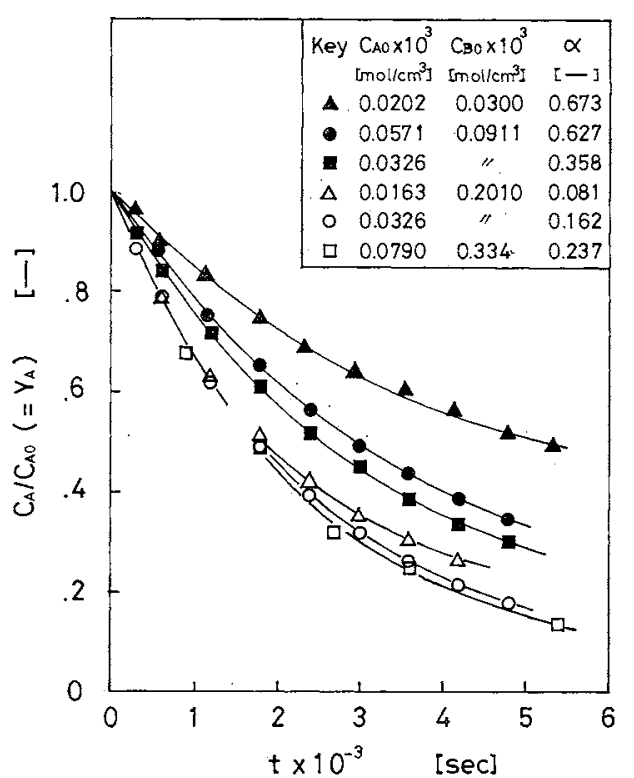

Fig. 5 Typical results of experiment

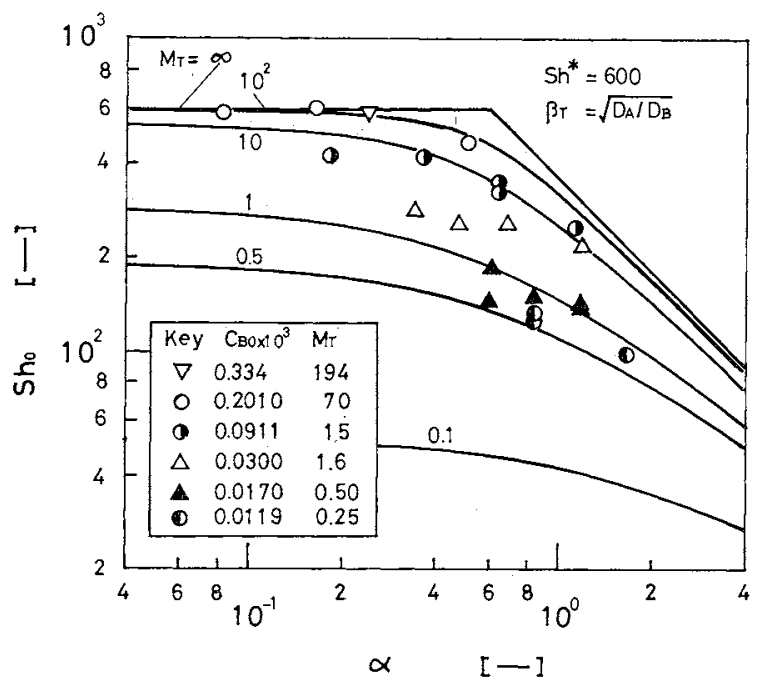

(a) $\beta_{T}=\sqrt{\overline{D_{A}}} / \overline{D_{B}}$

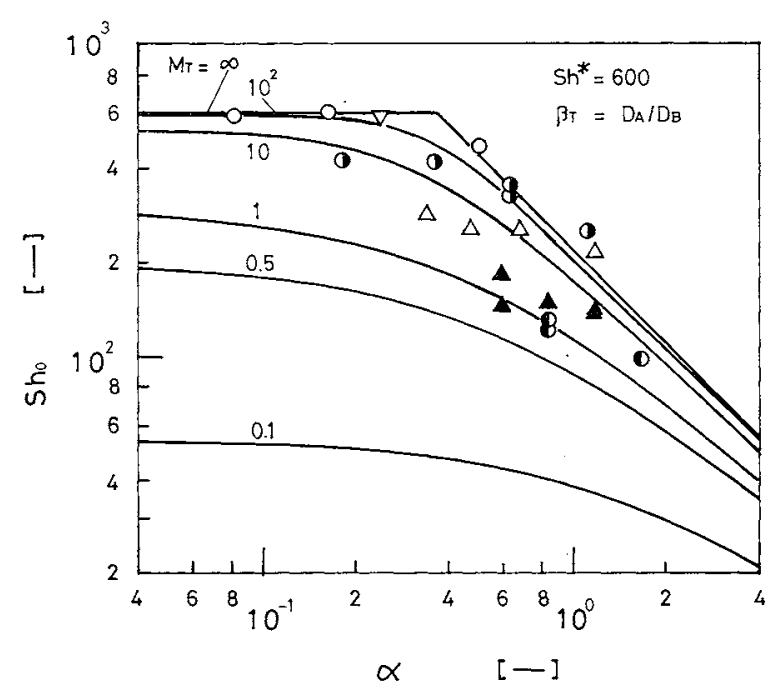

(b) $\beta_{T}=D_{A} / D_{B}$

Fig. 6 Correlation of extraction performance 
reaction with $\beta_{T}=\sqrt{ } D_{A} / D_{B}$ than with that where $\beta_{T}=D_{A} / D_{B}$. Although this is likely to correspond to the surface renewal model with equal fractional rates of renewal in both phases, further consideration is required. The data of much lower $C_{B 0}$ are rather deviated from the simulated lines: this may be ascribed presumably to the effects of reversible reaction or to the variation of interfacial rate process at extremely low $C_{B 0}$.

\section{Conclusion}

The extraction of hydrochloric acid by Amberlite LA-2 was carried out in a stirred transfer cell. It was found from the analysis of the initial rate of extraction that the interfacial rate process was significant in the range of amine concentration lower than $0.1 \mathrm{~mol} / l$, while in the range higher than $0.2 \mathrm{~mol} / l$ the interfacial rate process was assumed to be an instantaneous irreversible reaction. These experimental results were interpreted by a diffusion model that takes account of the interfacial rate process expressed by $(1,2)$ order irreversible reaction respect to the interfacial concentrations of hydrochloric acid and amine.

\section{Nomenclature}

$\begin{array}{llr}C & =\text { concentration } & {\left[\mathrm{mol} / \mathrm{cm}^{3}\right]} \\ D & =\text { diffusion coefficient } & {\left[\mathrm{cm}^{2} / \mathrm{sec}\right]} \\ k & =\text { mass transfer coefficient } & {\left[\mathrm{cm}^{2} / \mathrm{sec}\right]} \\ k_{r} & = & \text { interfacial rate constant } \\ M_{T} & = & \text { dimensionless reaction rate constant } \\ & & \left(=k_{r} C_{B 0}^{m+n-1} / k_{A}\right)\end{array}$

$\begin{array}{rlr}V & = & \text { volume of liquid phase } \\ v & \text { ratio of liquid volume }\left(=V_{A} / V_{B}\right) \\ \alpha & =\end{array}$

〈Subscripts〉

$A \quad=$ hydrochloric acid or aqueous phase

$B \quad=$ amine or organic phase

$i \quad=$ at the interface

$\langle$ Superscripts〉

$m \quad=$ order of reaction with respect to hydrochloric acid

$n \quad=$ order of reaction with respect to amine

\section{Literature Cited}

1) Chang, D. P. and C. R. Wilke: J. Phys. Chem., 59, 592 (1955).

2) Harada, M., H. Araki and W. Eguchi: Kagaku Kōgaku, 35, 1136 (1971).

3) Inoue, K., Y. Kawano, F. Nakashio and W. Sakai: ibid., 38, 41 (1971).

4) Inoue, K. and F. Nakashio: Kagaku Kogaku Ronbunshu, 1, 616 (1975).

5) Kakihana, H. and K. Narita: "Saishin no Ion Kokan", Hirokawa Shoten (1960).

6) Kataoka, T., T. Nakagawa and K. Ueyama: Chem. Eng. J., 10, 189 (1975).

7) Kataoka, T., T. Nishiki, T. Nakagawa and K. Ueyama: Kagaku Kögaku, 35, 1157 (1971).

8) Kawano, Y. and F. Nakashio: Kagaku Kogaku Ronbunshu, 3, 440 (1977).

9) Lewis, J. B.: Chem. Eng. Sci, 3, 248 (1954).

10) Sakai, W., F. Nakashio, T. Tsuneyuki and K. Inoue: Kagaku Kögaku, 33, 1221 (1969).

11) Schiffer, D. K., A. Hochhouser, D. F. Evans and E. L. Cussler: Nature, 250, 484 (1974).

12) Tsuneyuki, T. and F. Nakashio: unpublished.

(Presented in part at the 42th Annual Meeting of The Soc. of Chem. Engrs., Japan, at Hiroshima, 1977) 